\title{
Posicionamento sobre Uso de Antiplaquetários e Anticoagulantes nos Pacientes Infectados pelo Novo Coronavírus (COVID-19) - 2020
}

Position Statement on the Use of Antiplatelet Agents and Anticoagulants in Patients Infected with the New Coronavirus (COVID-19) - 2020

Realização: Grupo de Estudos de Antitrombóticos (GEAT) do Departamento de Cardiologia Clínica (DCC) da Sociedade Brasileira de Cardiologia (SBC)

Conselho de Normatizações e Diretrizes (2020-2021): Brivaldo Markman Filho, Antonio Carlos Sobral Sousa, Aurora Felice Castro Issa, Bruno Ramos Nascimento, Harry Correa Filho, Marcelo Luiz Campos Vieira

Coordenador de Normatizações e Diretrizes (2020-2021): Brivaldo Markman Filho

Autores do Posicionamento: Alexandre de Matos Soeiro, ${ }^{1,2}{ }^{\circledR}$ Tatiana de Carvalho Andreucci Torres Leal, ${ }^{1}$ Marcel de Paula Pereira, ${ }^{1,2}$ Eduardo Gomes Lima, ${ }^{1,3}$ Ana Cristina Baptista da Silva Figueiredo, ${ }^{4}$ João Luiz Fernandes Petriz, ${ }^{5}$ Dalton Betolim Precoma, ${ }^{6,7 ® 0}$ Carlos Vicente Serrano Jr. ${ }^{10}$

Instituto do Coração (Incor), Hospital das Clínicas da Faculdade de Medicina da Universidade de São Paulo (HCFMUSP), ${ }^{1}$ São Paulo, SP - Brasil Hospital BP Mirante, ${ }^{2}$ São Paulo, SP - Brasil

Hospital 9 de Julho, ${ }^{3}$ São Paulo, SP - Brasil

Hospital Glória D'Or, ${ }^{4}$ Rio de Janeiro, $R J-$ Brasil

Hospital Barra D'Or, ${ }^{5}$ Rio de Janeiro, RJ - Brasil

Sociedade Hospitalar Angelina Caron, ${ }^{6}$ Curitiba, $P R-$ Brasil

Pontifícia Universidade Católica do Paraná, ${ }^{7}$ Curitiba, PR - Brasil

Esta diretriz deverá ser citada como:

Soeiro AM, Leal TCAT, Pereira MP, Lima EG, Figueiredo ACBS, Petriz JLF, et al. Posicionamento sobre Uso de Antiplaquetários e Anticoagulantes nos Pacientes Infectados pelo Novo Coronavírus (COVID-19) - 2020. Arq Bras Cardiol. 2020; 115(2):292-301.

Nota: Estes posicionamentos se prestam a informar e não a substituir o julgamento clínico do médico que, em última análise, deve determinar o tratamento apropriado para seus pacientes.

Correspondência: Sociedade Brasileira de Cardiologia - Av. Marechal Câmara, 360/330 - Centro - Rio de Janeiro - CEP: $20020-907$. E-mail: diretrizes@cardiol.br 
Declaração de potencial conflito de interesses dos autores/colaboradores do Posicionamento do Departamento de Ergometria, Exercício, Cardiologia Nuclear e Reabilitação Cardiovascular (DERC/SBC) sobre a Atuação Médica em suas Áreas Durante a Pandemia por COVID-19

Se nos últimos 3 anos o autor/colaborador do Posicionamento:

\begin{tabular}{|c|c|c|c|c|c|c|c|}
\hline $\begin{array}{l}\text { Nomes Integrantes do } \\
\text { Posicionamento }\end{array}$ & $\begin{array}{l}\text { Participou de } \\
\text { estudos clínicos e/ } \\
\text { ou experimentais } \\
\text { subvencionados } \\
\text { pela indústria } \\
\text { farmacêutica ou } \\
\text { de equipamentos } \\
\text { relacionados ao } \\
\text { posicionamento } \\
\text { em questão }\end{array}$ & $\begin{array}{l}\text { Foi palestrante } \\
\text { em eventos } \\
\text { ou atividades } \\
\text { patrocinadas } \\
\text { pela indústria } \\
\text { relacionados ao } \\
\text { posicionamento em } \\
\text { questão }\end{array}$ & $\begin{array}{c}\text { Foi (é) } \\
\text { membro do } \\
\text { conselho } \\
\text { consultivo } \\
\text { ou diretivo } \\
\text { da indústria } \\
\text { farmacêutica } \\
\text { ou de } \\
\text { equipamentos }\end{array}$ & $\begin{array}{l}\text { Participou } \\
\text { de comitês } \\
\text { normativos } \\
\text { de estudos } \\
\text { científicos } \\
\text { patrocinados } \\
\text { pela indústria }\end{array}$ & $\begin{array}{c}\text { Recebeu } \\
\text { auxílio } \\
\text { pessoal ou } \\
\text { institucional } \\
\text { da indústria }\end{array}$ & $\begin{array}{c}\text { Elaborou } \\
\text { textos } \\
\text { científicos em } \\
\text { periódicos } \\
\text { patrocinados } \\
\text { pela indústria }\end{array}$ & $\begin{array}{l}\text { Tem ações } \\
\text { da indústria }\end{array}$ \\
\hline Antônio Carlos Avanza Jr. & Não & Não & Não & Não & Não & Não & Não \\
\hline $\begin{array}{l}\text { Carlos Alberto Cordeiro } \\
\text { Hossri }\end{array}$ & Não & Não & Não & Não & Não & Não & Não \\
\hline $\begin{array}{l}\text { Carlos Alberto Cyrillo } \\
\text { Sellera }\end{array}$ & Não & Não & Não & Não & Não & Não & Não \\
\hline Gabriel Blacher Grossman & Não & Não & Não & Não & Não & Não & Não \\
\hline Lara Terra F. Carreira & Não & Não & Não & Não & Não & Não & Não \\
\hline Luiz Eduardo Fonteles Ritt & Não & Não & Não & Não & Não & Não & Não \\
\hline Luiz Eduardo Mastrocola & Não & Não & Não & Não & Não & Não & Não \\
\hline Mauricio Batista Nunes & Não & Não & Não & Não & Não & Não & Não \\
\hline Mauricio Milani & Não & Não & Não & Não & Não & Não & Não \\
\hline Nabil Ghorayeb & Não & Não & Não & Não & Não & Não & Não \\
\hline $\begin{array}{l}\text { Odilon Gariglio Alvarenga } \\
\text { de Freitas }\end{array}$ & Não & Não & Não & Não & Não & Não & Não \\
\hline $\begin{array}{l}\text { Pedro Ferreira de } \\
\text { Albuquerque }\end{array}$ & Não & Não & Não & Não & Não & Não & Não \\
\hline Romeu Sergio Meneghelo & Não & Não & Não & Não & Não & Não & Não \\
\hline Salvador Manoel Serra & Não & Não & Não & Não & Não & Não & Não \\
\hline Tales de Carvalho & Não & Não & Não & Não & Não & Não & Não \\
\hline William Azem Chalela & Não & Não & Não & Não & Não & Não & Não \\
\hline
\end{tabular}




\section{Posicionamento}

\begin{abstract}
Sumário
1. Introdução

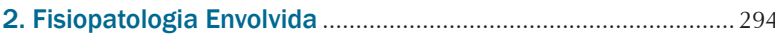

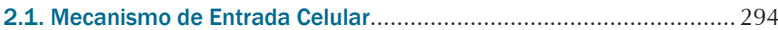

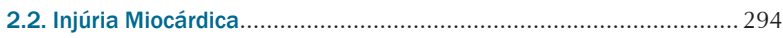

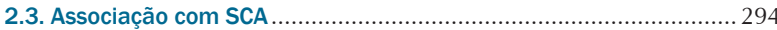

2.4. Mecanismo Tromboembólico ....................................................... 295

3. Interações Medicamentosas e Efeitos Cardiovasculares

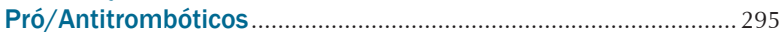

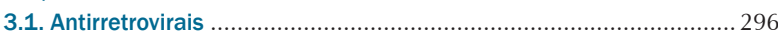

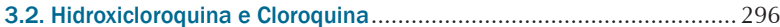

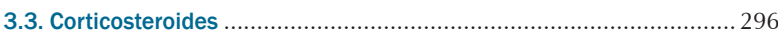

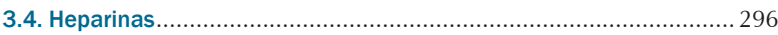

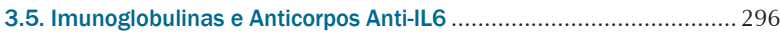

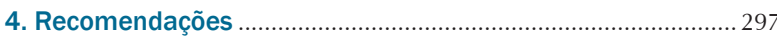

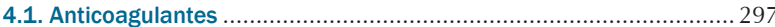

4.2. Antiplaquetários ......................................................................... 298

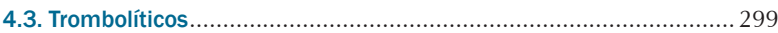

5. Considerações Finais ................................................................ 299

Referências ............................................................................. 300

\section{Introdução}

A pandemia pelo novo coronavírus (SARS-CoV-2) vem gerando debates a respeito do melhor tratamento para a doença e suas complicações. Publicações recentes demonstraram que as doenças cardiovasculares (DCV) estão entre os principais fatores de risco para evolução desfavorável da doença, incluindo hipertensão arterial e diabetes mellitus. ${ }^{1-6}$

Foi demonstrado que pacientes com infecção pelo novo coronavírus (COVID-19) apresentam mecanismos pró-trombóticos distintamente ativados, com maior possibilidade de eventos trombóticos ocorrerem. Síndrome coronariana aguda (SCA) com e sem supradesnivelamento do segmento ST pode ocorrer em pacientes com COVID-19, mas a real incidência ainda é incerta. ${ }^{7-10}$

Dessa forma, diversas questões relacionadas ao uso de medicamentos antiplaquetários e anticoagulantes em pacientes com suspeita ou infecção confirmada por COVID-19 permanecem incertas. Tais recomendações a seguir são válidas para as mais diversas situações clínicas como fibrilação atrial, síndrome coronariana aguda, doença arterial coronariana crônica, intervenção coronariana percutânea, pós-cirurgia cardíaca, acidente vascular encefálico (AVE) isquêmico e tromboembolismo venoso, devendo ser aplicadas caso-a-caso.

\section{Fisiopatologia Envolvida}

\subsection{Mecanismo de Entrada Celular}

$\mathrm{O}$ vírus SARS-CoV-2 tem como receptor funcional e porta de entrada a enzima conversora da angiotensina 2 (ECA2). Trata-se de uma carboxipeptidase que, diferente da enzima conversora da angiotensina 1 , tem efeito contrário, aumentando a degradação da angiotensina 2 e, portanto, apresenta efeito final vasodilatador. Além de estar presente no parênquima pulmonar, a ECA2 é distribuída também em todo sistema cardiovascular, rins e coração. Sabe-se que a ECA2 tem certa participação na função ventricular. Modelos animais que tem expressão reduzida de ECA2, apresentam disfunção ventricular esquerda severa. Aparentemente a infecção pelo novo coronavírus é capaz de promover downregulation desses receptores, o que poderia favorecer a injúria miocárdica e a lesão pulmonar., ${ }^{1,211}$. Apesar dessa possível associação, estudo observacional que avaliou 8910 pacientes infectados pelo SARS-Cov-2 não demonstrou aumento de mortalidade em pacientes em uso de medicações inibidores da ECA (IECA) e bloqueador de receptores de angiotensina (BRA). ${ }^{12}$ Desta forma, essas medicações devem ser mantidas em pacientes que faziam uso prévio e desenvolverem a infecção.

\subsection{Injúria Miocárdica}

Diversos estudos, principalmente chineses, vêm demonstrando o impacto da injúria miocárdica na instalação e progressão da COVID-19, assim como em sua apresentação de gravidade. Dados chineses descreveram a presença de injúria miocárdica em aproximadamente $20-30 \%$ dos pacientes internados, sendo presente também em 40\% naqueles que morreram. Há dados demonstrando que a elevações de alguns marcadores, como troponina e D-dímero, estão associados a pior prognóstico, com maior necessidade de internação em unidades de terapia intensiva, necessidade de ventilação mecânica e morte. ${ }^{2,13,14}$

O mecanismo da injúria miocárdica ainda não está bem estabelecido. Além do efeito direto do vírus, há o envolvimento do estresse miocárdico induzido pela falência respiratória e hipoxemia, com desbalanço de oferta e demanda, além de uma ação indireta da resposta inflamatória sistêmica sobre o tecido miocárdico e função endotelial. ${ }^{14-19}$

Não há dados suficientes sobre o efeito da inflamação miocárdica. Não se sabe ao certo se a miocardite induzida pela COVID-19 produza insuficiência cardíaca com fração de ejeção exclusivamente reduzida. Há relatos que descrevem achados anatomopatológicos de miocardite linfocítica, mesmo em pacientes com fração de ejeção preservada e sinais de hipertrofia ventricular. ${ }^{14-19}$

\subsection{Associação com SCA}

O risco aumentado para SCA em portadores da doença pode ser explicado pelo aumento da atividade trombótica descrita nesses pacientes, evidenciado pela frequente elevação do D-dímero e plaquetopenia. Além desse achado específico, sabese que há um aumento de eventos coronarianos em associação direta de infecções respiratórias virais. Fatores relacionados à atividade inflamatória, tais como disfunção endotelial, ativação plaquetária, ativação de macrófagos, disfunção hepática, expressão de fatores teciduais e liberação de citocinas são capazes de aumentar o risco de instabilização da placa aterosclerótica. Estudo mais recentes identificaram também níveis elevados de anticorpos antifosfolípides em pacientes com COVID-19, no entanto, não se sabe se isso apresenta alguma relação com gravidade da doença. ${ }^{5,7-10,15,20}$

No início da fase de infecção, a inibição plaquetária pode reduzir a formação de fibrina intravascular e trombos. Dessa forma, uso de aspirina pré-hospitalar, mas não o uso pósadmissão, foi associado menor risco para o desenvolvimento de insuficiência respiratória e mortalidade em pacientes com pneumonia adquirida pela comunidade. Em segundo lugar, 
a escolha de inibidores P2Y12 orais: apesar do fato de que todos os inibidores P2Y12 reduzem agregados de plaquetas e leucócitos e citocinas pró-inflamatórias derivadas de plaquetas, ticagrelor é único em ter um alvo adicional bem documentado de inibição, transportador nucleosídeo equilibrativo 1 (ENT1), contribuindo para a inibição da captação de adenosina celular. Portanto, ticagrelor confere propriedades anti-inflamatórias mais potentes, embora não testado em COVID-19.

Vale ressaltar que na vigência do quadro clínico infeccioso e, visto a alta incidência de injúria miocárdica decorrente a presença direta do vírus e de efeitos indiretos da infecção, mesmo na vigência de alterações eletrocardiográficas, o diagnóstico de SCA deve ser considerado frente à possibilidade de doença não isquêmica. Foi publicado ${ }^{21}$ relato de 18 casos de elevação do segmento ST em hospitais de Nova York, sugerindo SCA com supra de ST. Dentre eles, 4 tinham elevação difusa e 14 tinham elevações focais do segmento ST. $50 \%$ dos pacientes fizeram cineangiocoronariografia e 33\% não tinham doença coronariana obstrutiva. No total, 44\% dos pacientes tiveram diagnóstico de infarto agudo do miocárdio. Dessa forma, mesmo em pacientes com elevação do segmento de ST, diagnósticos diferenciais devem ser levantados, visto quadro clínico heterogêneo. Independente da etiologia, a mortalidade foi de $72 \%$. A decisão terapêutica e a estratificação invasiva deve ser ponderada levando em consideração quadro clínico, achados de exames complementares, experiência da equipe e disponibilidade do laboratório de hemodinâmica. ${ }^{14,16}$. Em outras duas publicações, demonstraram-se que houve menor incidência de SCA no norte da Califórnia, em relação ao mesmo período no ano anterior e que houve maior incidência de parada cardíaca extra-hospitalar, na Itália. Isto sugere uma menor procura da população aos serviços de emergência. ${ }^{22,23}$

Além de todos esses fatores, uma enorme preocupação tem sido levantada devido aos efeitos do isolamento social. A maioria das pessoas reduziram drasticamente a atividade física. Além disso, a alimentação por vezes torna-se inadequada com ingesta maior de carboidratos. Tais mudanças no estilo de vida podem ser fatores adicionais capazes de desencadear e contribuir com eventos trombóticos com AVE e SCA. ${ }^{24}$

\subsection{Mecanismo Tromboembólico}

Pacientes infectados pelo COVID-19 provavelmente apresentam risco aumentado de tromboembolismo venoso (TEV). Embora não haja nenhuma grande série de casos publicados até agora, há relatos de parâmetros anormais de coagulação em pacientes hospitalizados com doença COVID-19 grave. ${ }^{25-27}$

Estudo recente mostrou em uma série de 106 casos de COVID-19 submetidos à angiotomografia de artérias pulmonares na investigação de TEV pulmonar que $30 \%$ dos pacientes houve confirmação de tromboembolismo venoso. Pacientes com infecção por COVID-19 e embolia pulmonar apresentaram níveis mais elevados de D-dímero do que aqueles sem embolia $(p<0,001)$, além de maior necessidade de internação em terapia intensiva (75\% vs. $32 \%, p<0,001$ ). A presença de D-dímero $>2.660$ $\mu \mathrm{g} / \mathrm{L}$ apresentou sensibilidade de $100 \%$ e especificidade de $67 \%$ para embolia pulmonar. ${ }^{28}$

O D-dímero tem sido associado a maior taxa de mortalidade e parece aumentar progressivamente com a exacerbação da infecção. A fase da doença em que ocorre o desenvolvimento de síndrome do desconforto respiratório agudo (SDRA) e a piora do padrão radiológico é marcada pela elevação expressiva de D-dímero, observando-se nos casos mais graves injúria miocárdica e coagulação intravascular disseminada (CIVD). A resposta inflamatória sistêmica em pacientes com infecção pode resultar em lesão endotelial com consequente aumento na geração de trombina e redução da fibrinólise endógena. Esse estado prótrombótico é denominado coagulopatia induzida pela sepse (SIC) e precede a CIVD. Os diversos mecanismos envolvidos na SIC agem simultaneamente, culminando em um estado pró-hemostático. Aparentemente, os fatores mais importantes que medeiam esse distúrbio do sistema de coagulação durante a sepse são as citocinas inflamatórias. ${ }^{29}$

Evidências demonstram uma interação cruzada entre inflamação e coagulação, com a inflamação induzindo a ativação da coagulação e a coagulação acentuando a atividade inflamatória. As plaquetas têm um papel central no desenvolvimento das anormalidades da coagulação na sepse e podem ser ativadas diretamente por mediadores pró-inflamatórios, como o fator ativador de plaquetas, bem como por meio da trombina gerada. A ativação de plaquetas também pode estimular a formação de fibrina por mecanismo alternativo. A expressão de P-selectina na membrana plaquetária não apenas induz a adesão de plaquetas a leucócitos e células endoteliais, mas também aumenta a expressão do fator tecidual nos monócitos. Em circunstâncias normais, a ativação da coagulação é controlada por três importantes vias anticoagulantes fisiológicas: o sistema antitrombina, o sistema ativado da proteína $\mathrm{C}$ e o inibidor da via do fator tecidual. $\mathrm{Na}$ sepse, todas as três vias sofrem disfunção. Em meio a todo esse desbalanço do sistema de coagulação, a fibrinólise endógena é amplamente reduzida. ${ }^{29}$

Em um estudo de coorte retrospectiva da China, níveis elevados de D-dímero (>1g/L) foram fortemente associados ao óbito hospitalar. Em outro estudo comparando sobreviventes da COVID-19 com não-sobreviventes, os não sobreviventes tiveram níveis significativamente mais elevados de D-dímero e produtos de degradação de fibrinas e 71,4\% dos não sobreviventes atenderam aos critérios clínicos de CIVD durante o curso da doença. Além da CIVD, pacientes gravemente doentes com imobilização prolongada estão inerentemente em alto risco para TEV. A inflamação vascular também pode contribuir para o estado hipercoagulante e endotelial disfunção em tais pacientes. No cenário de pacientes COVID-19 gravemente doentes que demonstram clínico deterioração como evidenciado por hipóxia ou instabilidade hemodinâmica, doença tromboembólica deve ser considerada. ${ }^{25-27}$

\section{Interações Medicamentosas e Efeitos Cardiovasculares Pró/Antitrombóticos}

Até o momento, não há tratamento específico para a infecção pelo COVID-19 e um arsenal terapêutico vem sendo usado em situações de gravidade em ambiente hospitalar, muitos ainda em investigação de eficácia e segurança.

Como esses medicamentos podem ser usados em situações específicas, vale considerar seus efeitos colaterais sobre o sistema cardiovascular e possíveis interações medicamentosas com outras terapias frequentemente utilizadas em pacientes cardiopatas. 


\section{Posicionamento}

\subsection{Antirretrovirais}

A ribavirina, e o remdesivir são agentes que agem bloqueando a RNA polimerase e o lopinavir/ritonavir inibem a replicação viral.

Não há descrição de cardiotoxicidade induzido pela ribavirina. Por sua vez, o lopinavir/ritonavir produz alargamento do intervalo QT e PR, principalmente em pacientes que já apresentam QT longo ou estão em uso de outras drogas que também interagem sobre o intervalo QT. Tanto a ribavirina quanto o lopinavir/ritonavir potencializam o efeito anticoagulante, modificando a ação da varfarina (principalmente ribavirina) ou novos anticoagulantes como apixabana e rivaroxabana (principalmente lopinavir-ritonavir). ${ }^{30-32}$

Em outro estudo, o uso de dabigatrana em pacientes internados por COVID-19 com uso de antivirais, teve aumento dos níveis séricos plasmáticos, com necessidade de retirada da droga em mais da metade dos pacientes. ${ }^{31}$

O lopinavir-ritonavir podem também influenciar na atividade dos inibidores P2Y12 por inibição da CYP3A4 o que reduz o nível sérico de metabólitos ativos do clopidogrel e aumenta a atividade do ticagrelor. Assim, pelo alto risco de sangramento, o uso concomitante de ticagrelor e lopinavir-ritonavir deve ser desencorajado. ${ }^{11}$

Há também evidência de que o uso de clopidogrel na vigência de tratamento com lopinavir-ritonavir possa produzir efeito antiagregante insuficiente, o que não é observado com prasugrel, sendo, portanto, a droga mais ideal ${ }^{32} \mathrm{Na}$ presença de contraindicações para o uso de prasugrel (tais como AVE prévio, idade avançada, índice de massa corpórea baixa e risco aumentado de sangramento) indica-se o uso de clopidogrel, sendo sugerido a realização de avaliação de atividade plaquetária. ${ }^{30,32}$

Remdesivir é um antirretroviral em investigação para COVID-19 e que já foi utilizado durante a epidemia de Ebola. Apesar de medicação promissora, em estudo randomizado, duplo-cego, multicêntrico, a prescrição de remdesivir não resultou em desfechos de mortalidade em relação ao placebo. Houve tendência a diminuição de sintomas, porém sem significância estatística. ${ }^{33}$ Não há descrições sobre cardiotoxicidade e outras interações medicamentosas importantes até o momento. .0,32 $^{30}$

\subsection{Hidroxicloroquina e Cloroquina}

Hidroxicloroquina e cloroquina são medicações frequentemente utilizadas em pacientes portadores de malária e outras doenças inflamatórias sistêmicas tais como lúpus eritematoso sistêmico e artrite reumatoide. Aparentemente, são capazes bloquear a entrada de vírus pelas células, além de produzir imunomodulação, atenuando a produção de citocinas, a inibição de autofagia e atividade lisossomal no hospedeiro. Podem exercer propriedades antitrombóticas, especialmente contra anticorpos antifosfolípides. 5,34,35

Há evidências anteriores do uso e de sua eficácia em epidemias prévias no tratamento de SARS e MERS. Um estudo chinês demonstrou que em 100 pacientes infectados pelo COVID-19, o uso de cloroquina foi relacionado a melhora do padrão radiológico, maior depuração viral e menor progressão da doença. Apesar dos resultados promissores, o estudo apresenta diversas limitações e tem diversos vieses de interpretação. ${ }^{5,34,35}$
Em outras publicações de estudos observacionais, demonstrouse que o uso de hidroxicloroquina associado ou não ao uso de azitromicina não resultou em desfechos favoráveis. Não houve diminuição de mortalidade ou tempo de intubação, assim como não houve diferença de soroconversão em pacientes com doença leve a moderada. ${ }^{36-38}$ Diversos estudos sobre o impacto da hidroxicloroquina e/ou cloroquina estão em andamento.

Apesar de classicamente bem toleradas, a cloroquina e a hidroxicloroquina podem induzir graves efeitos colaterais tais como aumentar o intervalo QT, induzir hipoglicemia, retinopatia e distúrbios neuropsiquiátricos. No entanto, não há descrição de interação com medicamentos antiplaquetários ou anticoagulantes. ${ }^{5,34,35}$

\subsection{Corticosteroides}

Metilprednisolona é outra droga cujo uso pode ser considerado durante a apresentação grave de COVID-19 e síndrome do desconforto respiratório agudo. Sabidamente provoca retenção hídrica, alterações hidroeletrolíticas e hipertensão. Porém, também não há descrição de interação com medicamentos antiplaquetários ou anticoagulantes. ${ }^{35}$

\subsection{Heparinas}

Em estudo chinês ${ }^{39}$ com 449 pacientes internados por COVID-19, mostrou-se que a estratégia de prescrição de enoxaparina 40-60mg/dia ou heparina não-fracionada $10.000 \mathrm{a}$ 15.000U/dia trouxe benefícios de mortalidade em 28 dias em 2 subgrupos. Um deles era de pacientes com critérios de $\mathrm{SIC} \geq 4$ (que usa os critérios de aumento de TP, queda na contagem de plaquetas e aumento do SOFA-Score), com diferença de $40 \%$ vs. $64,2 \%$ ( $p=0,029$ ) e o outro subgrupo era composto por pacientes com D-dímero $>6 x$ o limite da normalidade, com diferença de $32,8 \%$ vs. $52,4 \%$ ( $p=0,017)$, demonstrando que a estratégia de prescrição de profilaxia química de TEV ou anticoagulação plena devem ser consideradas individualmente em todos os pacientes com COVID-19 internados, assim como a pesquisa de eventos trombóticos deve ser buscada com maior intensidade. ${ }^{26,27,31,39-41}$

\subsection{Imunoglobulinas e Anticorpos Anti-IL6}

A lógica por trás do uso da imunoglobulina depende do uso dos mecanismos: neutralização viral e imunomodulação. Uma aplicação intrigante do mecanismo anterior é o uso de soro convalescente ou plasma. Tal terapia tem efeitos pleiotrópicos que culminam na supressão da inflamação e, portanto, esta terapia pode potencialmente aliviar a gravidade da doença na fase de hiperinflamação. Evidências mais robustas são necessárias para confirmar esses achados. Da mesma forma, há uma boa razão para se perguntar se os pacientes com COVID-19 com tempestade de citocinas podem se beneficiar de anticorpos monoclonais direcionados ao receptor de interleucina 6(IL-6) que foram bem-sucedidos em atenuar a inflamação em pacientes transplantados. Talvez isso se reflita no contexto trombótico do paciente, porém ainda não há evidências concretas sobre isso. ${ }^{15}$

Anticorpos anti-IL 6 aumentam a expressão da CYP3A4. No entanto, não existe nenhuma recomendação de ajuste de dose de antiplaquetários ou anticoagulantes em pacientes em uso dessa terapia. 


\section{Recomendações}

\subsection{Anticoagulantes}

A prescrição de heparina de baixo peso molecular ou heparina não-fracionada profiláticas para TEV ou como anticoagulação plena devem ser individualizadas, sendo sempre consideradas em pacientes de alto risco de TEV internados. ${ }^{5,29,39-43}$

A terapia anticoagulante em pacientes com COVID-19 grave e indícios de SIC e/ou com D-dímero muito elevado em associação a outros biomarcadores que denotam gravidade, na ausência de contraindicação à anticoagulação, pode ser considerada uma estratégia terapêutica fundamentada no consenso de especialistas e em poucos estudos retrospectivos. Adicionalmente, essa estratégia requer a utilização de protocolos institucionais rígidos que permitam a vigilância e a rápida intervenção frente a complicações. ${ }^{29}$

É possível que a terapêutica anticoagulante seja mais benéfica quando iniciada na fase pré-trombótica do que nos quadros avançados, quando o risco de sangramento é maior. Em se optando pela anticoagulação, parece razoável o uso de heparina de baixo peso molecular como fármaco de escolha em pacientes estáveis e com depuração normal de creatinina (dose de $1 \mathrm{mg} / \mathrm{kg}$ de 12/12h, subcutânea). Em caso de choque ou depuração de creatinina abaixo de $50 \mathrm{ml} / \mathrm{min} / \mathrm{m}^{2}$, é preferível o uso de heparina nãofracionada intravenosa $(18 \mathrm{UI} / \mathrm{kg} / \mathrm{h})$, tendo como alvo um tempo de tromboplastina parcial ativada entre 1,5 e 1,8. Entretanto, não há evidências que fundamentem a ampla utilização de heparina em dose terapêutica na COVID-19. A Figura 1 ilustra uma sugestão de avaliação e estratégia terapêutica nesse grupo de pacientes baseado nas poucas evidências vigentes. ${ }^{29}$
Já a European Society of Cardiology propôs uma recomendação de realizar anticoagulação plena em todos os pacientes com sinais de gravidade como: aumento da frequência respiratória $>24$ ipm, saturação arterial de oxigênio < 90\%, proteína-C reativa elevada, níveis elevados de D-dímero ou em ascensão e níveis de fibrinogênio elevados. Especificamente em relação ao D-dímero a orientação é de anticoagulação plena quando > $3.000 \mathrm{ng} /$ $\mathrm{mL}$, somente profilaxia química quando $<500 \mathrm{ng} / \mathrm{mL}$ e uso de enoxaparina $40 \mathrm{mg}$ de 12/12 h quando D-dímero estiver entre 500 e $3.000 \mathrm{ng} / \mathrm{mL}^{42}$

A manutenção de profilaxia para tromboembolismo após a alta hospitalar também deve ser individualizada com heparina de baixo peso molecular ou novos anticoagulantes, pesando risco benefício de eventos trombóticos versus sangramento (Figura 2).

Enquanto não há evidência específica para COVID-19, é razoável considerar individualizar a estratificação de risco de eventos tromboembólicos e hemorrágicos e estender a profilaxia medicamentosa por até 45 dias, considerando pacientes com mobilidade reduzida, câncer, TEV prévio, D-dímero elevado > 2 vezes o limite superior. ${ }^{5}$

Em relação aos pacientes que já fazem uso de anticoagulação prévia por quaisquer motivos, a medicação deve ser mantida sempre que possível. Caso o paciente seja internado por sintomas de pneumonia por COVID-19 a manutenção da medicação deve ser individualizada. Em pacientes graves pode-se ter alterações na farmacocinética das medicações, insuficiência renal, insuficiência hepática, plaquetopneia e CIVD, e a anticoagulação parenteral com uso de heparina de baixo peso molecular ou heparina não-fracionada deve ser preferencial, caso não haja contraindicações. 5, , 26, 27,31,39,40

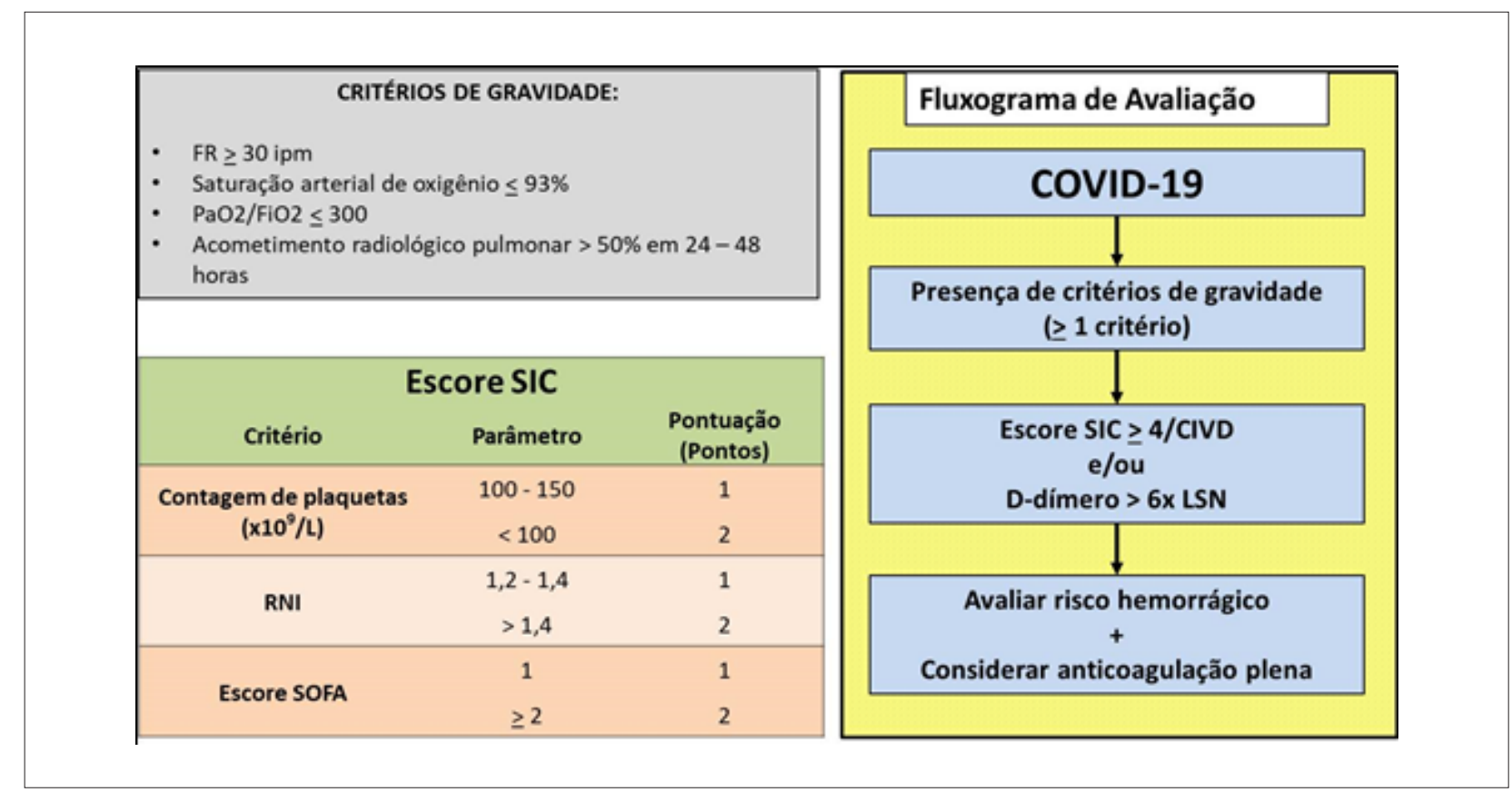

Figura 1 - Sugestão de manejo e fluxograma para avaliação de terapia anticoagulante em pacientes graves com COVID-19.

FR: frequência respiratória; SIC: coagulopatia induzida pela sepse; RNI: razão de normatização internacional; SOFA: sequential organ failure assessment; CIVD: coagulação intravascular disseminada; LSN: limite superior da normalidade. 


\section{Posicionamento}

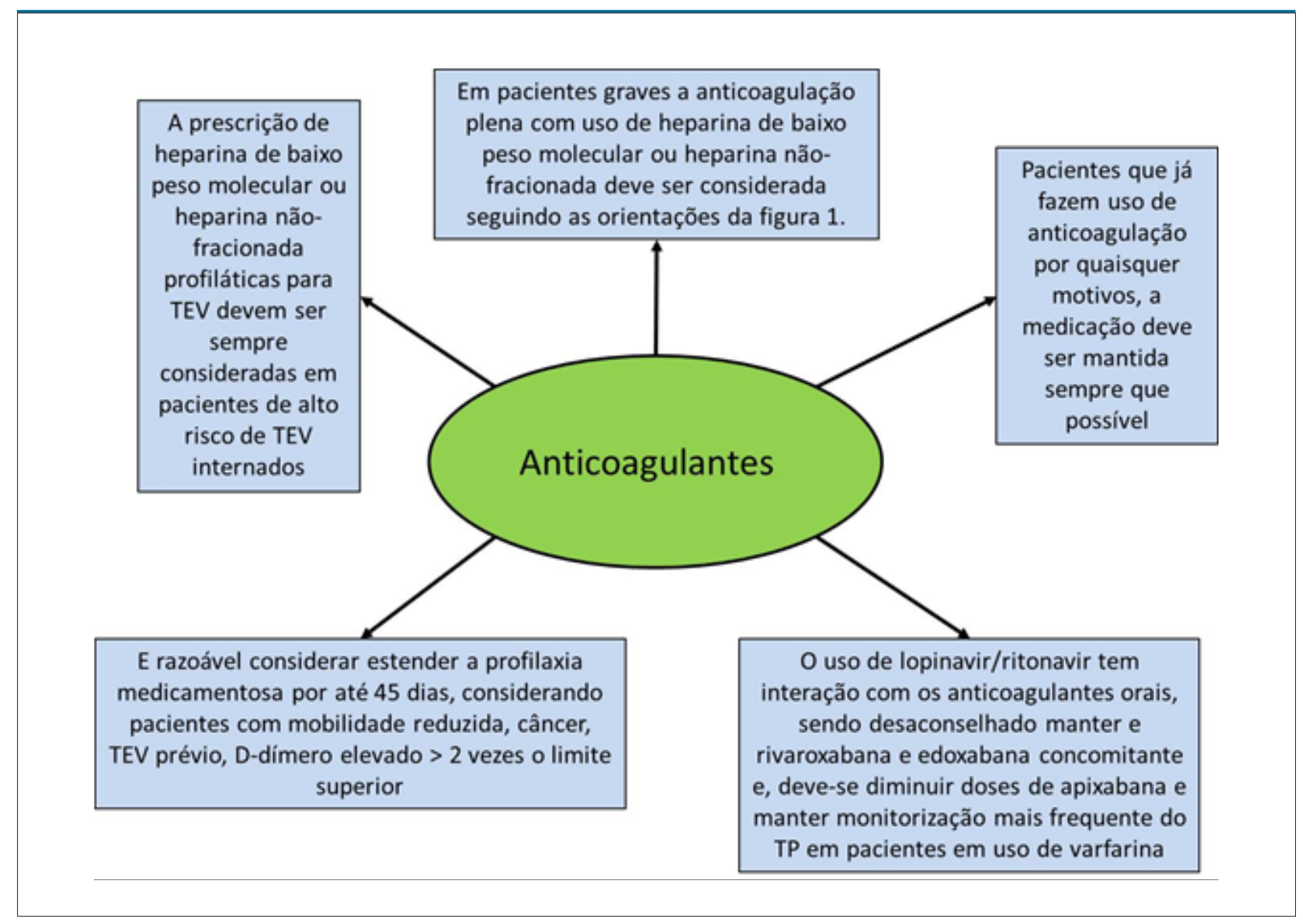

Figura 2 - Recomendação quanto ao uso de anticoagulantes em pacientes com infecção por COVID-19.

TEV: tromboembolismo venoso; TP: tempo de protrombina.

O uso de lopinavir/ritonavir tem interação com os anticoagulantes orais, sendo desaconselhado manter e rivaroxabana e edoxabana concomitante e, diminuir doses de apixabana e monitorização mais frequente do TP em pacientes em uso de varfarina. Dessa forma, em pacientes com uso prévio de anticoagulação oral e que necessitam manter a medicação na internação, aconselha-se trocar pela forma parenteral por heparina de baixo peso molecular. É válido lembrar que caso os pacientes evoluam com discrasia sanguínea/CIVD deve-se pesar o risco de sangramento ao manter essas medicações, sendo necessária a suspensão na quase totalidade dos casos. ${ }^{32}$

\subsection{Antiplaquetários}

Em relação ao uso de antiplaquetários, pacientes que fazem uso dessas medicações no cenário de doença coronariana crônica, a medicação deve ser mantida. Em pacientes que usam dupla antiagregação plaquetária, deve-se individualizar a prescrição de tais medicações em pacientes internados (Figura 3). 5,7,40

Dado o alto risco de sangramento em pacientes após intervenção coronariana percutânea (ICP) complicada pela COVID-19, a menor duração da dupla antiagregação plaquetária (DAPT) pode ser benéfica nesta população, além do uso preferencial do clopidogrel naqueles com risco de sangramento elevado, pesando-se sempre o risco de trombose de stent vs. sangramento. Para contrabalancear o aumento do risco de hemorragia associado à DAPT, estudos mais recentes forneceram evidências que suportam a suspensão precoce de aspirina após ICP, reduzindo principalmente taxas de sangramento. Entre os pacientes em uso de DAPT, manter o inibidor P2Y12 em monoterapia (preferencialmente ticagrelor) pode ser uma estratégia razoável após ICP realizada há mais de 3 meses. Devido à falta de evidências, para aqueles com ICP realizada há menos de 3 meses, DAPT não deve ser descontinuada. ${ }^{5,7}$

Caso o paciente necessite do uso de antivirais, há interação entre lopinavir/ritonavir com clopidogrel e ticagrelor, devendo-se evitar essas medicações ou avaliar atividade plaquetária. Prasugrel pode ser administrado com cautela, salvo contra-indicações inerentes ao medicamento. Não há descrição de interações com antiplaquetários endovenosos como cangrelor. ${ }^{11,30,32}$

Consensos de especialistas recomendam medidas proativas ou mesmo parar toda a terapia antiplaquetária em pacientes com uma contagem de plaquetas $<100.000 / \mu \mathrm{L}$ e $<50.000 /$ $\mu \mathrm{L}$, respectivamente. ${ }^{7}$ Há entretanto recomendação mais atual que sugere reduzir para monoterapia se $<50.000 / \mu \mathrm{L}$ e suspender caso fique $<25.000 / \mu \mathrm{L}$, considerando sempre o risco isquêmico vs. risco de sangramento. ${ }^{5}$ 


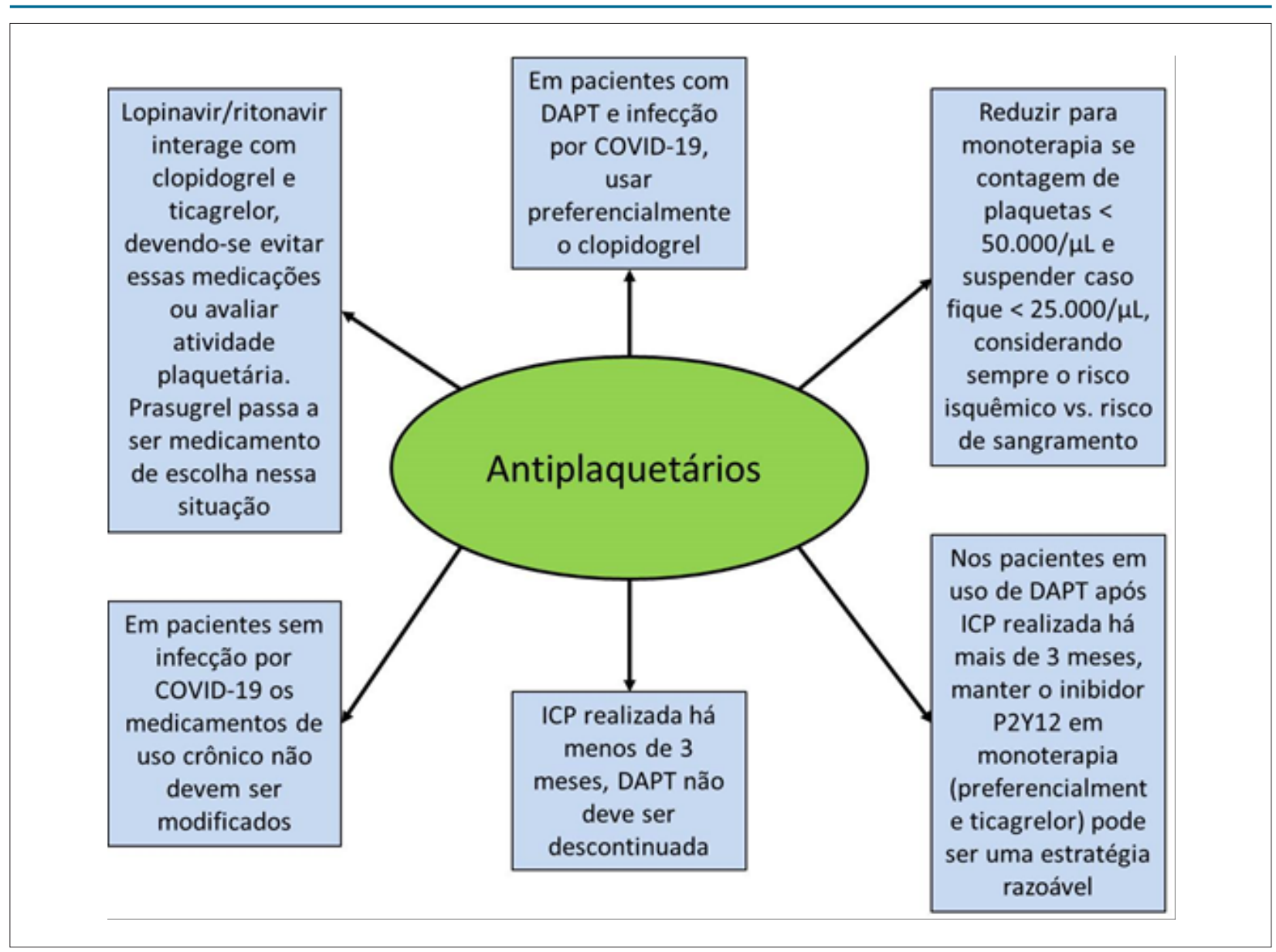

Figura 3 - Recomendação quanto ao uso de antiplaquetários em pacientes com infecção por COVID-19. ICP: intervenção coronariana percutânea; DAPT: dupla antiagregação plaquetária.

\subsection{Trombolíticos}

Tanto a American Heart Association quanto a European Society of Cardiology indicam o uso de trombólise como primeira opção em pacientes suspeitos/confirmados com COVID-19 e infarto agudo do miocárdio com supradesnivelamento do segmento ST, principalmente em centros sem serviço de hemodinâmica ou naqueles com hemodinâmica disponível, porém sem preparo adequado para evitar a contaminação da equipe envolvida. ${ }^{5,7,44-47}$

Dessa forma, não existe até o momento nenhuma contraindicação ao uso de trombolíticos nesse contexto e seu uso deve ser pautado de acordo com as contra-indicações usuais, sendo individualizado em situações de instabilidade elétrica ou hemodinâmica, CIVD, plaquetopenia, sangramentos e insuficiência renal ou hepática. ${ }^{48}$
Vale ressaltar que diagnósticos diferenciais de supradesnivelamento do segmento ST devem ser sempre aventados, como miopericardite, nos quais a trombólise deve ser evitada. ${ }^{5}$

\section{Considerações Finais}

As evidências a respeito da COVID-19 e suas interações com os sistemas de coagulação e ativação plaquetária ainda são iniciais. Existem fortes indícios de que essa via possa ser um alvo terapêutico importante. No entanto, ainda são necessários estudos mais robustos para determinar a real importância dos mecanismos pró-trombóticos e a melhor terapia a ser adotada nesse grupo de pacientes. 


\section{Posicionamento}

Tabela 1 - Recomendações gerais para sobre Covid-19/antiplaquetários e anticoagulantes

\begin{tabular}{|c|c|c|}
\hline Indicação & Classe de Recomendação & Nível de Evidência \\
\hline \multicolumn{3}{|l|}{ Associação medicamentosa entre terapias antritrombóticas e medicações utilizadas no tratamento do COVID-19 } \\
\hline Em pacientes em uso de Lopinavir-Ritonavir o Prasugrel deve ser o antiplaquetário de escolha & $\| B$ & B \\
\hline Em pacientes em uso de Lopinavir-Ritonavir, caso contra-indicado Prasugrel, deve-se escolher Clopidogrel & IIB & B \\
\hline Em pacientes em uso de Lopinavir-Ritonavir, caso optado pelo Clopidogrel, a monitorização de atividade plaquetária & $\| \mathrm{B}$ & B \\
\hline Em pacientes em uso de Lopinavir-Ritonavir, o uso de Ticagrelor deve ser desencorajado & $\| B$ & B \\
\hline $\begin{array}{l}\text { Em pacientes em uso de anticoagulação prévia e que farão uso de Lopinavir-Ritonavir, deve-se trocar o } \\
\text { anticoagulante pela forma parenteral (Heparinas) }\end{array}$ & $\| \mathrm{A}$ & B \\
\hline $\begin{array}{l}\text { Em pacientes em uso de anticoagulação prévia, evitar associação de Rivaroxabana ou Edoxabana com } \\
\text { Lopinavir-Ritonavir }\end{array}$ & III & B \\
\hline $\begin{array}{l}\text { Em pacientes em uso de anticoagulação prévia com varfarina, com necessidade de manutenç̧ão desta medicação e } \\
\text { estiverem em uso de Lopinavir-Ritonavir, deve-se avaliar com maior frequência o TP }\end{array}$ & $\| \mathrm{A}$ & B \\
\hline O uso de Remdesevir não tem interação medicamentosa importante com antiplaquetários e anticoagulantes & $\| \mathrm{A}$ & B \\
\hline O uso de corticosteróides não tem interação medicamentosa importante com antiplaquetários e anticoagulantes & $\| \mathrm{A}$ & B \\
\hline $\begin{array}{l}\text { Imunoglobulinas e anticorpos anti-IL6 não tem interação medicamentosa importante com antiplaquetários } \\
\text { e anticoagulantes }\end{array}$ & $\| B$ & B \\
\hline $\begin{array}{l}\text { O uso de Hidroxicloroquina ou Cloroquina não tem interação medicamentosa importante com antiplaquetários } \\
\text { e anticoagulantes }\end{array}$ & $\| B$ & B \\
\hline Em pacientes que fizerem uso de Hidroxicloroquina ou Cloroquina, deve-se monitorizar o intervalo QT & IA & B \\
\hline \multicolumn{3}{|l|}{ Uso de anticoagulantes em pacientes infectados pelo COVID-19 } \\
\hline Profilaxia química para eventos tromboembólicos deve ser instituída em todos pacientes internados & $\| \mathrm{A}$ & B \\
\hline $\begin{array}{l}\text { A anticoagulação plena deve ser considerada em casos especiais, pesando risco-benefícios, como por exemplo, } \\
\text { utilizando o escore de coagulopatia induzida por sepse ou D-Dímero > 6x o limite superior da normalidade }\end{array}$ & $\| \mathrm{B}$ & B \\
\hline Em pacientes que fazem uso de anticoagulação anteriormente, a medicação deve ser mantida sempre que possível & $\| \mathrm{A}$ & B \\
\hline Considerar estender profilaxia química para eventos tromboembólicos até 45 dias após a alta em pacientes de risco & $\| \mathrm{B}$ & B \\
\hline \multicolumn{3}{|l|}{ Uso de antiplaquetários em pacientes infectados pelo COVID-19 } \\
\hline Em pacientes que faziam uso no cenário de doença coronariana crônica, a medicação deve ser mantida & $\| \mathrm{A}$ & C \\
\hline $\begin{array}{l}\text { Em pacientes em uso de dupla antiagregação no cenário pós angioplastia (ATC), pode-se considerar manter } \\
\text { em monoterapia os pacientes com ATC com duração maior que } 3 \text { meses, pesando o risco de sangramento e } \\
\text { trombose de stent }\end{array}$ & $\| A$ & B \\
\hline
\end{tabular}

\section{Referências}

1. Guan WJ, Ni ZY, Hu Y, Liang WH, Ou CQ, He JX, et al. Clinical characteristics of coronavirus disease 2019 in China. N Engl J Med. 2020; 382(18):1708-20.

2. Zhou F, Yu T, Du R, Fan G, Liu Y, Liu Z, et al. Clinical course and risk factors for mortality of adult inpatients with COVID-19 in Wuhan, China: a retrospective cohort study. Lancet. 2020; 395(10229):1054-62.

3. Li B, Yang J, Zhao F, Zhi L, Wang X, Liu L, et al. Prevalence and impact of cardiovascular metabolic diseases on COVID-19 in China. Clin Res Cardiol. 2020; 109(5):531-8.

4. Wang D, Hu B, Hu C, Zhu F, Liu X, Zhang J, et al. Clinical characteristics of 138 hospitalized patients with 2019 novel coronavirus-infected pneumonia in Wuhan, China. JAMA. 2020; 323(11):1061-9.

5. Bikdeli B, Madhavan MV, Jimenez D, Chuich T, Dreyfus I, Driggin E, et al. COVID-19 and thrombotic or thromboembolic disease: implications for prevention, antithrombotic therapy, and follow-up. J Am Coll Cardiol. 2020 Apr 15; pii:S0735-1097(20)35008-7.

6. Costa IBSS, Bittar CS, Rizk SI, Araújo Filho AEA, Santos KAQ, Machado TIV, et al. The heart and COVID-19: what cardiologists need to know. Arq Bras Cardiol. 2020; 114(5):805-16.
7. Zhou X, Li Y, Yang O. Antiplatelet therapy after percutaneous coronary intervention in patients with COVID-19: implications from clinical features to pathologic findings. Circulation. 2020; 141(22):1736-8.

8. Seyoum M, Enawgaw B, Melku M. Human blood platelets and viruses: defense mechanismand role in the removal of viral pathogens. ThrombJ. 2018Jul 17; 16:16.

9. Antoniak $\mathrm{S}$. The coagulation system in host defense. The coagulation system in host defense. Res Pract Thromb Haemost. 2018; 2(3):549-57.

10. Goeijenbier M, van Wissen M, van de Weg C, Jong E, Gerdes VE, Meijers JC, et al. Review: viral infections and mechanisms of thrombosis and bleeding. JMed Virol. 2012; 84(10):1680-96.

11. Driggin E, Madhavan MV, Bikdeli B, Chuich T, Laracy J, Bondi-Zoccai G, et al. Cardiovascular considerations for patients, health care workers, and health systems during the coronavirus disease 2019 (COVID-19) pandemic. J Am Coll Cardiol. 2020; 75(18):2352-71.

12. Mehra MR, Desai SS, Kuy S, Henry TD, Patel AN. Cardiovascular disease, drug therapy, and mortality in Covid-19. N Engl J Med. 2020May 1. [Epubahead of print].

13. Lippi G, Lavie CJ, Sanchis-Gomar F. Cardiac troponin I in patients with coronavirus disease 2019 (COVID-19): evidence from a meta-analysis. Prog Cardiovasc Dis. 2020 Mar 10. [Epub ahead of print]. 
14. Shi S, Qin M, Shen B, Cai Y, Liu T, Yang F, et al. Association of cardiac injury with mortality in hospitalized patients with COVID-19 in Wuhan, China. JAMA Cardiol. 2020 Mar 25:e200950. [Epub ahead of print].

15. Akhmerov A, Marbán E. COVID-19 and the Heart. Circ Res. 2020; 126(10):1443-55

16. Ruan Q, Yang K, Wang W, Jiang L, Song J. Clinical predictors of mortality due to COVID- 19 based on an analysis of data of 150 patients from Wuhan, China. Intensive Care Med. 2020 Mar 03; 46:845-8.

17. Guo T, Fan Y, Chen M, Wu X, Zhang L, He T, et al. Cardiovascular implications of fatal outcomes of patients with coronavirus disease 2019 (COVID-19). JAMA Cardiol. 2020 Mar 27. [Epub ahead of print].

18. Peiris JSM, Chu CM, Cheng VCC, Chan KS, Hung IFN, Poon LLM, et al. Clinical progression and viral load in a community outbreak of coronavirus-associated SARS pneumonia: a prospective study. Lancet. 2003; 361(9371):1767-72.

19. Inciardi RM, Lupi L, Zaccone G, Italia L, Raffo M, Tomasoni D, et al. Cardiac involvement in a patient with coronavírus disease 2019 (COVID-19). JAMA Cardiol. 2020 Mar 27. [Epub ahead of print].

20. Corrales-Medina VF, Madjid M, Musher DM. Role of acute infection in triggering acute coronary syndromes. Lancet Infect Dis. 2010; 10(2):83-92.

21. Bangalore S, Sharma A, Slotwiner A, Yatskar L, Harari R, Shah B, el al. STsegment elevation in patients with Covid-19- a case series. N Engl J Med. 2020 Apr 17. [Epub ahead of print]

22. Solomon MD, McNulty EJ, Rana JS, Leong TK, Lee C, Sung S, et al. The Covid-19 pandemic and the incidence of acute myocardial infarction. N Engl J Med. 2020 May 19. [Epub ahead of print].

23. Baldi E, Sechi GM, Mare C, Canevari F, Brancaglione A, Primi R, et al. Outof-hospital cardiac arrest during the Covid-19 outbreak in Italy. N Engl J Med. 2020 Apr 29. [Epub ahead of print]

24. G, Henry BM, Sanchis-Gomar F. Physical inactivity and cardiovascular disease at the time of coronavirus disease 2019 (COVID-19). Eur I Prev Cardiol. 2020; 27(9):906-8.

25. Kochi AN, Tagliari AP, Forleo GB, Fassini GM, Tondo C. Cardiac and arrhythmic complications in patients with Covid-19. J Cardiovasc Electrophysiol. 2020; 31(5):1003-8.

26. Cui S, Chen S, Li X, Liu S, Wang F. Prevalence of venous thromboembolism in patients with severe novel coronavirus pneumonia. J Thromb Haemost. 2020; 18(6):1421-4

27. Dolhnikoff M, Duarte-Neto AN, Almeida Monteiro RA, Ferraz da Silva LF, Pierre de Oliveira E, Saldiva PHN, et al. Pathological evidence of pulmonary thrombotic phenomena in severe COVID-19. J Thromb Haemost. 2020; 18(6):1517-9.

28. Leonard-Lorant I, DelabrancheX, Severac F, Helms J, Pauzet C, Collange O, et al. Acute pulmonary embolism in COVID-19 patients on CT angiography and relationship to D-Dimer levels. Radiology. 2020 Apr 23; 201561. [Epub ahead of print].

29. Nascimento JHP, Gomes BFO, Resende P, Petriz JLF, Rizk SI, Costa IBSS, et al. COVID-19 and hypercoagulable state: a new therapeutic perspective. Arq Bras Cardiol. 2020; 114(5):823-7.

30. Itkonen MK, Tornio A, Lapatto-Reiniluoto O, Neuvonen M, Neuvonen PJ, Niemi $M$, et al. Clopidogrel increases dasabuvir exposure with or without ritonavir, and ritonavir inhibits the bioactivation of clopidogrel. Clin Pharmacol Ther. 2019; 105(1):219-28

31. Testa S, Prandoni P, Paoletti O, Morandini R, Tala M, Dellanoce C, et al. Direct oral anticoagulant plasma levels striking increase in severe COVID-19 respiratory syndrome patients treated with antiviral agents: the cremona experience. J Thromb Haemost. 2020; 18(6):1320-3.
32. Marsousi N, Daali Y, Fontana P, Reny J, Ancrenaz-Sirot V, Calmy A, et al Impact of boosted antiretroviral therapy on the pharmacokinetics and efficacy of clopidogrel and prasugrel active metabolites. Clin Pharmacokinet. 2018; 57(10):1347-54.

33. Wang Y, Zhang D, Du G, Du R, Zhao J, Jin Y, et al. Remdesivir in adults with severe COVID-19: a randomised, double-blind, placebo-controlled, multicentre trial. Lancet. 2020; 395(10236):1569-78.

34. Gao J, Tian Z, Yang X. Breakthrough: chloroquine phosphate has shown apparent efficacy in treatment of COVID-19 associated pneumonia in clinical studies. Biosci Trends. 2020; 14(1):72-3.

35. Sanders JM, Monogue ML, Jodlowski TZ, Cutrell JB. Pharmacologic treatments for coronavirus disease 2019 (COVID-19): a review. JAMA. 2020 323(18)1824-36.

36. Geleris J, Sun Y, Platt J, Zucker J, Baldwin M, Hripcsak G, et al. Observational study of hydroxychloroquine in hospitalized patients with Covid-19. N Eng J Med. 2020 May 7. [Epub ahead of print].

37. Rosenberg ES, Dufort EM, Udo T, Wilberschied LA, Kumar I, Tesoriero J, et al. Association of treatment with hydroxychloroquine or azithromycin with in-hospital mortality in patients with COVID-19 in New York State. JAMA. 2020 May 11:e208630. [Epub ahead of print].

38. Tang W, Cao Z, Han M, Wang Z, Chen J, Sun W, et al. Hydroxychloroquine in patients with mainly mild to moderate coronavirus disease 2019: open label, randomised controlled trial. BMJ. 2020 May 14. [Epub ahead of print].

39. Tang N, Bai H, Chen X, Gong J, Li D, Sun Z. Anticoagulant treatment is associated with decreased mortality in severe coronavirus disease 2019 patients with coagulopathy. J Thromb Haemost. 2020; 18(5):1094-9.

40. Vivas D, Rold 'an V, Esteve-Pastor MA, Rold'an I, Tello-Montoliu A, RuizNodar JM, et al. Recomendaciones sobre el tratamento antitrombotico durante la pandemia COVID-19. Posicionamiento del Grupo de Trabajo de Trombosis Cardiovascular de la Sociedad Espanola de Cardiologra. Rev Esp Cardiol. 2020 Apr 22. [Epub ahead of print].

41. Pereira MP, Lima EG, Serrano Júnior CV. Viral infections and atherothrombosis: another caution in the wake of COVID-19? Rev Assoc Med Bras. 2020; 66(3):366-9.

42. Bassam Atallah B, Mallah SI, AlMahmeed W. Anticoagulation in COVID-19. Eur Heart J Cardiovasc Pharmacother. 2020 Apr 30. [Epub ahead of print].

43. Thachil J, Tang N, Gando S, Falanga A, Cattaneo M, Levi M, et al. ISTH interim guidance on recognition and management of coagulopathy in COVID-19.J Thromb Haemost. 2020; 18(5):1023-26.

44. European Society of Cardiology. ESC Guidance for the Diagnosis and Management of CV Disease during the COVID-19 Pandemic; 2020. [citado 28 May 2020]. Disponível em: https://www.escardio.org/Education/COVID19-and-Cardiology/ESCCOVID-19-Guidance.

45. Mahmud E, Dauerman HL, Welt FG, Messenger JC, Rao SV, Grines C et al. Management of acute myocardial infarction during the COVID-19 pandemic: A Consensus Statement From the Society for Cardiovascular Angiography and Interventions (SCAI), the American College of Cardiology (ACC), and the American College of Emergency Physicians (ACEP). Catheter Cardiovasc Interv. 2020 Apr 20. [Epub ahead of print].

46. Abdelaziz HK, Patel B, Chalil S, Choudhury T. COVID-19 pandemic and acute myocardial infarction: management protocol from a British Cardiac Centre. Crit Pathw Cardiol. 2020; 19(2):55-7.

47. Tan W, Aboulhosn J. The cardiovascular burden of coronavirus disease 2019 (COVID-19) with a focus on congenital heart disease. Int J Cardiol. 2020 Jun 15; 309:70-7.

48. Zeng J, Huang I, Pan L. How to balance acute myocardial infarction and COVID-19: the protocols from Sichuan Provincial People's Hospital. Intensive Care Med. 2020 Mar 11;1-3. [Epub ahead of print]. 